\title{
IMAge Retrieval USING BIT-Plane PIXEL DISTRIBUTION
}

\author{
N S T Sai ${ }^{1}$ and R C Patil ${ }^{2}$ \\ ${ }^{1}$ Tech Mahindra Limited, Mumbai, India. \\ nstsai@techmahindra.com \\ ${ }^{2}$ Mukesh Patel School of Tech. Mgmt. and Engineering, SVKM's, NMIMS University \\ ravindra.patilethakureducation.org
}

\section{ABSTRACT}

Users needing is to store and index the image data and retrieved the image on feature vector derived by the user. Content Based Image Retrieval (CBIR) is search engine to retrieving the desired image automatically from the large image database having different categories. Retrieving the relevant images from the database by using feature vector is the challenging and important task. It is also need to retrieve the images from variety of the domain that is the application of CBIR that domains are medicine, crime prevention ,Biometrics, architecture, Fashion and publishing. This paper present the method developed to search and retrieve the similar image using bit plane image. Bit plane images are formed by using threshold and using bit plane slicing. Mean, standard deviation and third moment of row and column pixel distribution of bit plane image is used as a feature vector. We use simple Euclidean distance to compute the similarity measures of images for Content Based Image Retrieval application. The average precision and average recall of each image category and over all precision and recall is considered for the performance measure.

\section{KEYWORDS}

CBIR, bit plane, precision, recall, mean, standard deviation, moment.

\section{INTRODUCTION}

Now a day due to modern technology there is need to efficient storage of large no. of digital images. The term CBIR seems to have originated in 1992, when it was used by T. Kato to describe experiments into automatic retrieval of images from a database, based on the colors and shapes present. Since then, the term has been used to describe the process of retrieving desired images from a large collection on the basis of syntactical image features. The techniques, tools and algorithms that are used originate from fields such as statistics, pattern recognition, signal processing, and computer vision. Historians from a variety of disciplines art, sociology, medicine, etc. - use visual information sources to support their research activities. Archaeologists also rely heavily on images. In some instances (particularly, but not exclusively, art), the visual record may be the only evidence available. Where access to the original works of art is restricted or impossible, perhaps due to their geographic distance, ownership restrictions or factors to do with their physical condition, researchers have to use surrogates in the form of photographs, slides or other pictures of the objects, which may be collected within a particular library, museum or art gallery. Photographic and slide collections are maintained by a wide range of organizations, including academic and public libraries.

CBIR draws many of its methods from the field of image processing and computer vision, and is regarded by some as a subset of that field. It differs from these fields principally through its emphasis on the retrieval of images with desired characteristics from a collection of significant size. Image processing covers a much wider field, including image enhancement, compression, transmission, and interpretation. While there are grey areas (such as object recognition by feature analysis), the distinction between mainstream image analysis and CBIR is usually fairly 
clear-cut. An example may make this clear. Many police forces now use automatic face recognition systems. Such systems may be used in one of two ways. Firstly, the image in front of the camera may be compared with a single individual's database record to verify his or her identity. In this case, only two images are matched, a process few observers would call CBIR. Secondly, the entire database may be searched to find the most closely matching images. This is a genuine example of CBIR.

Research and development issues in CBIR cover a range of topics, many shared with mainstream image processing and information retrieval. Some of the most important are:

- Understanding image users' needs and information-seeking behavior

- Identification of suitable ways of describing image content

- $\quad$ Extracting such features from raw images

- $\quad$ providing compact storage for large image databases

- Matching query and stored images in a way that reflects human similarity judgments

- $\quad$ Efficiently accessing stored images by content

- $\quad$ Providing usable human interfaces to CBIR systems.

\section{TEXT BASED IMAge ReTRIEVAL}

Current indexing practice for images relies largely on text descriptors or classification codes, supported in some cases by text retrieval packages designed or adapted specially to handle images. Again, remarkably little evidence on the effectiveness of such systems has been published. User satisfaction with such systems appears to vary considerably. Text based image retrieval required manual annotation for every image. Describing every image by using the text string is very time consuming task. Text string describe the content of image that dependent on the user. For image perception of different user are different so text string for same image by different user will be different. So there is need of Content Based Image Retrieval. Contentbased Image Retrieval (CBIR) is fast growing technology and is the field that deals with application of computer vision for retrieval of images from digital libraries.

\section{CONTENT BaSed IMAge ReTrieval}

Content-based image retrieval (CBIR), also known as query by image content (QBIC) and content-based visual information retrieval (CBVIR) is the application of computer vision to the image retrieval problem, that is, the problem of searching for digital images in large databases. "Content-based" means that the search will analyse the actual contents of the image. The term 'content' in this context might refer to colors, shapes, textures, or any other information that can be derived from the image itself. Without the ability to examine image content, searches must rely on metadata such as captions or keywords, which may be laborious or expensive to produce. The need to find a desired image from a collection is shared by many professional groups, including journalists, design engineers and art historians.

There are to tasks in the Content Based Image Retrieval first is the population phase and second is retrieval phase. As shown in the Fig. 1 in the population phase feature vector (signature) extracted for every image in the database. This feature vector accurately represents the image. Feature vector size should be smaller than the image. Make a feature vector database for all the images. In the second task take a query image compute the feature vector and find out 
the Euclidean distance (similarity).The images whose minimum Euclidean distance can be retrieved [3], [13], [14],[15].

Bit plane slicing is well known technique used in Image processing. In image compression Bit plane slicing is used. Bit plane slicing is the conversion of image into multilevel binary image. These binary images are then compressed using different algorithm [7][8]. Each pixel is the series of digital bits from these digital bits binary images is to be extracted. Extracted binary image gives us binary information. This information is used for computing image feature in the image retrieval.

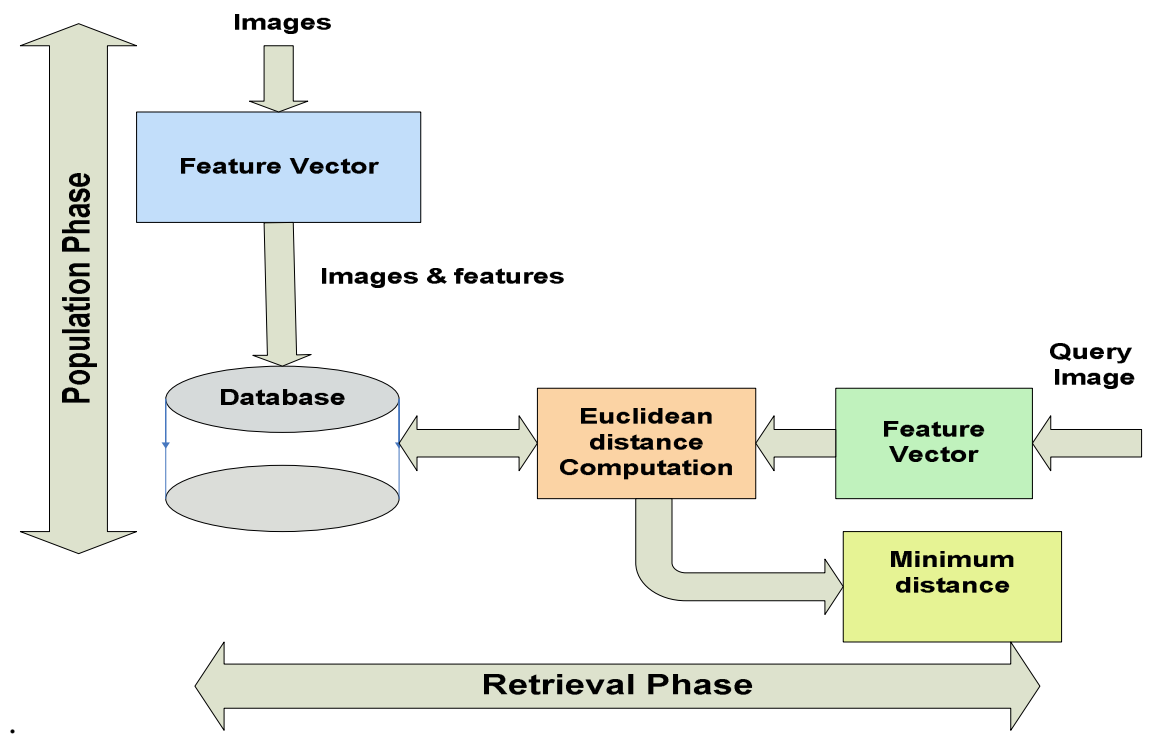

Figure 1. Block diagram of Content Based Image Retrieval.

\section{THEORETICAL BACKGROUND}

Color image or gray scale image is represented as a M X N intensity matrix, where each element have different intensity value which is lying [0-255] for gray scale image and it varies for the color image. Each intensity value of each pixel in the image can be represented by "1" or "0" single bit which is also known as binary image or an 8-bit. Given image is converted into the bit plane image (binary image) using different techniques $[1,2,3]$.

\subsection{Single Bit Plane Formation using Interband Average Image}

Single bit plane image is formed by using threshold .This threshold is mean of the image intensity value. First we consider the interband average image[9],[3],[4]. This inter band average image is formed by taking average of red, green and blue color planes. Let color image $I(i, j)$ having size $256 \times 256$. Then $r(i, j), g(i, j)$ and $b(i, j)$ are the three red, green and blue color plane for the given image respectively. $\operatorname{IBAI}(i, j)$ is the interband average image having size $256 \times 256$ and which is calculated as

$$
\operatorname{IBAI}(i, j)=\frac{1}{3} \sum_{i=1}^{256} \sum_{j=1}^{256} r(i, j)+g(i, j)+b(i, j)
$$

Then mean $M$ value of this interband average image is calculated as 
International Journal of Computer Science \& Information Technology (IJCSIT), Vol 3, No 3, June 2011

$$
M=\frac{1}{(256 X 256)} \sum_{i=1}^{256} \sum_{j=1}^{256} \operatorname{IBAI}(i, j)
$$

This mean value is considered as a threshold. Then single bit plane is created by comparing the mean value which is given above equation 2 with the each pixel value in the interband average image is greater than equal to the threshold then the corresponding pixel position of single bit plane will have a value of 1 otherwise it will have value of 0 .Created $\operatorname{SBP}(i, j)$ image is single bit plane image having the same size that of the original image.

$$
\operatorname{SBP}(i, j)=\left\{\begin{array}{lll}
1 & \text { if } & \operatorname{IBAI}(i, j) \geq M \\
0 & \text { if } & \operatorname{IBAI}(i, j)<M
\end{array}\right.
$$

Figure. 2 shows given image, interband average image for the same image and single bit plane $\operatorname{SBP}(i, j)$ for interband average image.

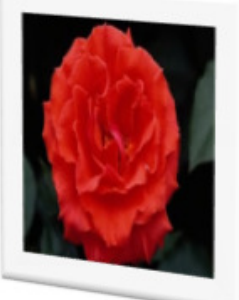

Given image

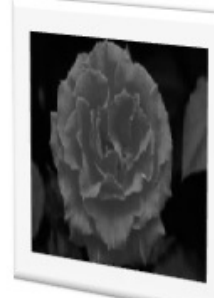

Interband average image

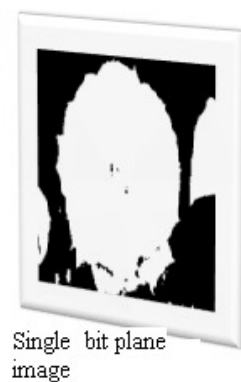

image

Figure 2. Single bit plane formation using interband average for given image

\subsection{Three Bit Plane Formation using Three Color Plane}

Well known color spaces considered commonly by all the researches are Red, Green and Blue. Mostly color images are stored in the RGB color format. In this method the color image is divide into R, G, and B components. Then compute three thresholds which is the mean of each color components $M R, M G, M B$ for red, green and blue color component respectively given as

$$
\begin{aligned}
& M R=\frac{1}{256 X 256} \sum_{i=1}^{256} \sum_{j=1}^{256} r(i, j) \\
& M G=\frac{1}{256 X 256} \sum_{i=1}^{256} \sum_{j=1}^{256} g(i, j) \\
& M B=\frac{1}{256 X 256} \sum_{i=1}^{256} \sum_{j=1}^{256} b(i, j)
\end{aligned}
$$

Here three bit planes will be computed as BMR, BMG and BMB. 


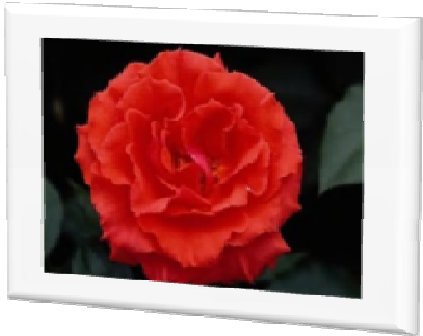

Given Rose image

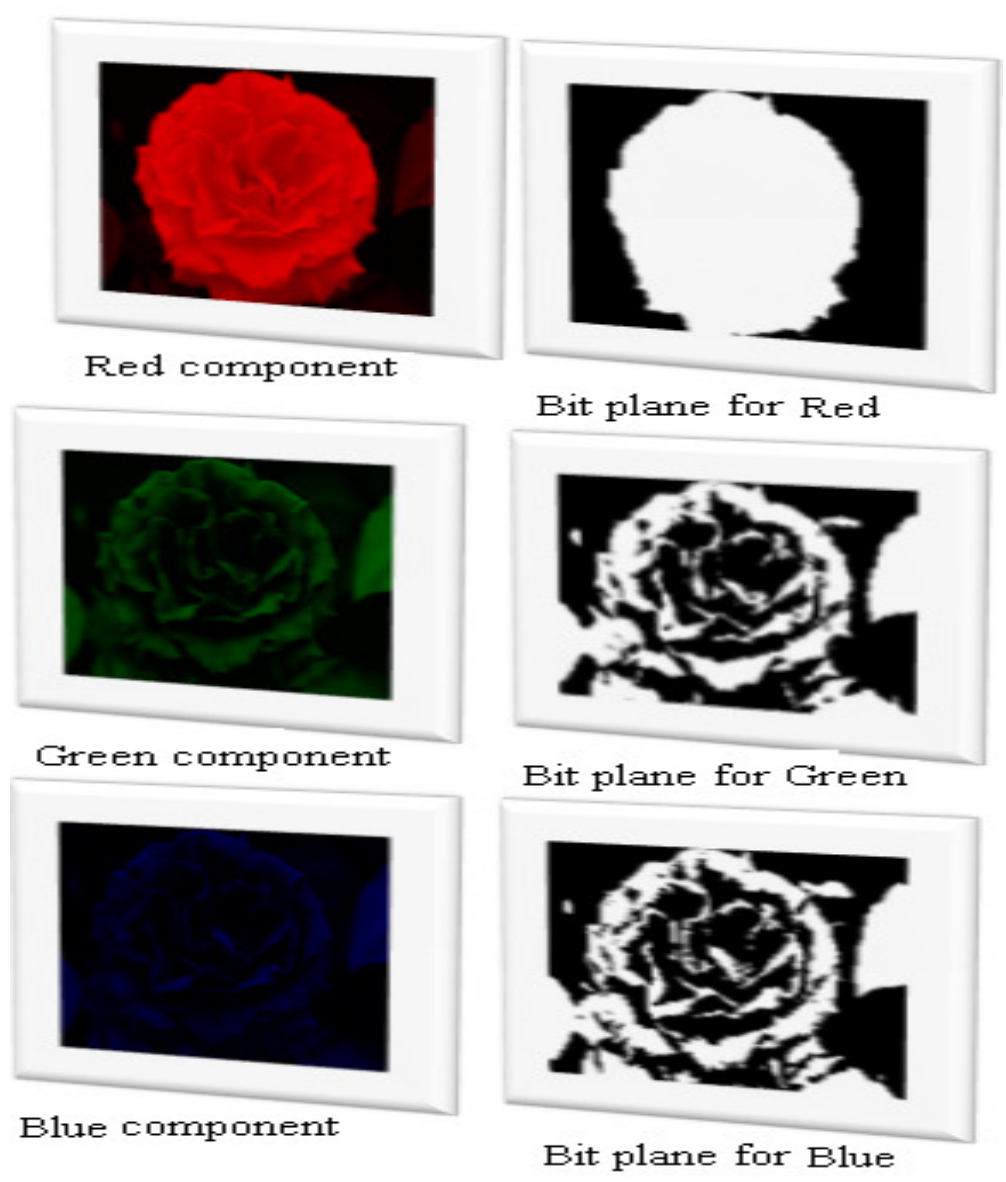

Figure 3.Three bit plane formation using red, green and blue plane for given image.

If a pixel in each component ( $R, G$ and $B)$ is greater than or equal to the respective threshold, the corresponding pixel position of the bit plane will have a value of 1 otherwise it will have a value of 0 .

$$
B M R(i, j)=\left\{\begin{array}{lll}
1 & \text { If } & r(i, j)>=M R \\
0 & \text { If } & r(i, j)<M R
\end{array}\right.
$$


International Journal of Computer Science \& Information Technology (IJCSIT), Vol 3, No 3, June 2011

$$
\begin{aligned}
& B M G(i, j)=\left\{\begin{array}{lll}
1 & \text { If } & g(i, j)>=M G \\
0 & \text { If } & g(i, j)<M G
\end{array}\right. \\
& B M B(i, j)=\left\{\begin{array}{lll}
1 & \text { If } & b(i, j)>=M B \\
0 & \text { If } & b(i, j)<M B
\end{array}\right.
\end{aligned}
$$

Figure. 3 shows given color image R, G and B components for color image and bit plane $B M R(i, j)$ for red component , bit plane $B M G(i, j)$ for green component and bit plane $B M B(i, j)$ for green component for given color image.

\subsection{Bit Plane Slicing}

Low contract of image enhanced by the image enhancement method .But this method brighten all the pixels of given image so this drawback is overcome in the bit-plane sclicing. In Bit-Plane Slicing image is sliced into eight binary planes[7]. The bits which are presents in the bit plane 0 is the least significant bit and the bits which are present in the bit plane 7 are the most significant bits as shown in Fig.4.

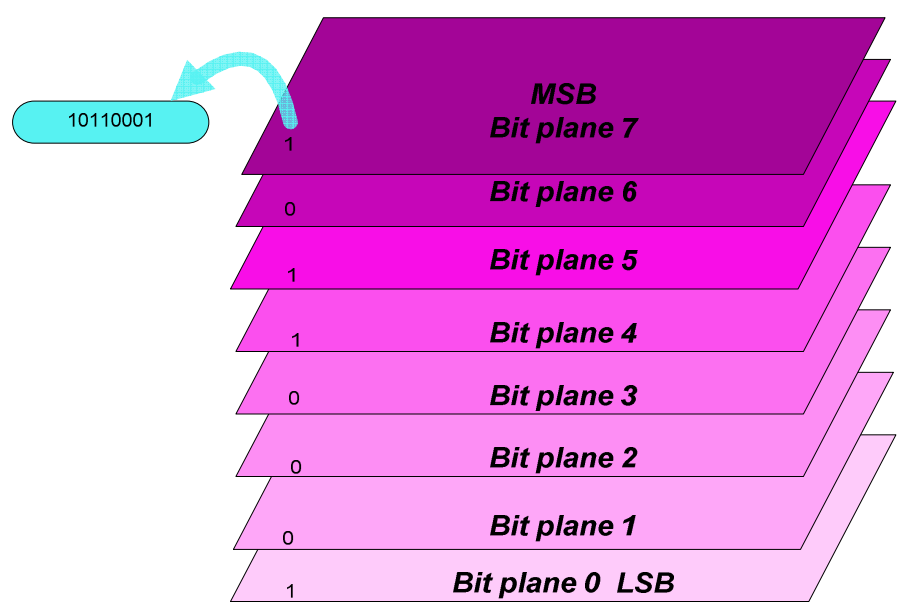

Figure 4. Bit plane Slicing.

It is clear that the intensity value of each pixel can be represented by an 8-bit binary vector $(b 7, b 6, b 5, b 4, b 3, b 2, b 1, b 0)$, where $\mathrm{i}$ is from 0 to 7 and each $b i$ is either " 0 " or" 1 ". In this case, an image may be considered as an overlay of eight bit-planes. Each bit-plane can be thought of as a twotone image and can be represented by a binary matrix .

The formation of bit plane is given

$$
\mathrm{I} b p(i, j)=R\left\{\frac{1}{2} \text { floor }\left[\frac{1}{2^{i}} I(i, j)\right]\right\}
$$

where $I(i, j)=$ original image,

$\mathrm{I} b p(i, j)=$ bit-plane information,

$\mathrm{R}=$ remainder and

floor $(i)=$ round the elements to i nearest integers less than or equal to $\mathrm{i}$. 
For each gray scale image we are getting 8 bit planes. Thus if we separate out R, G and B color components and create 8 bit planes for each color component then we can create total twenty four bit planes for the color image.

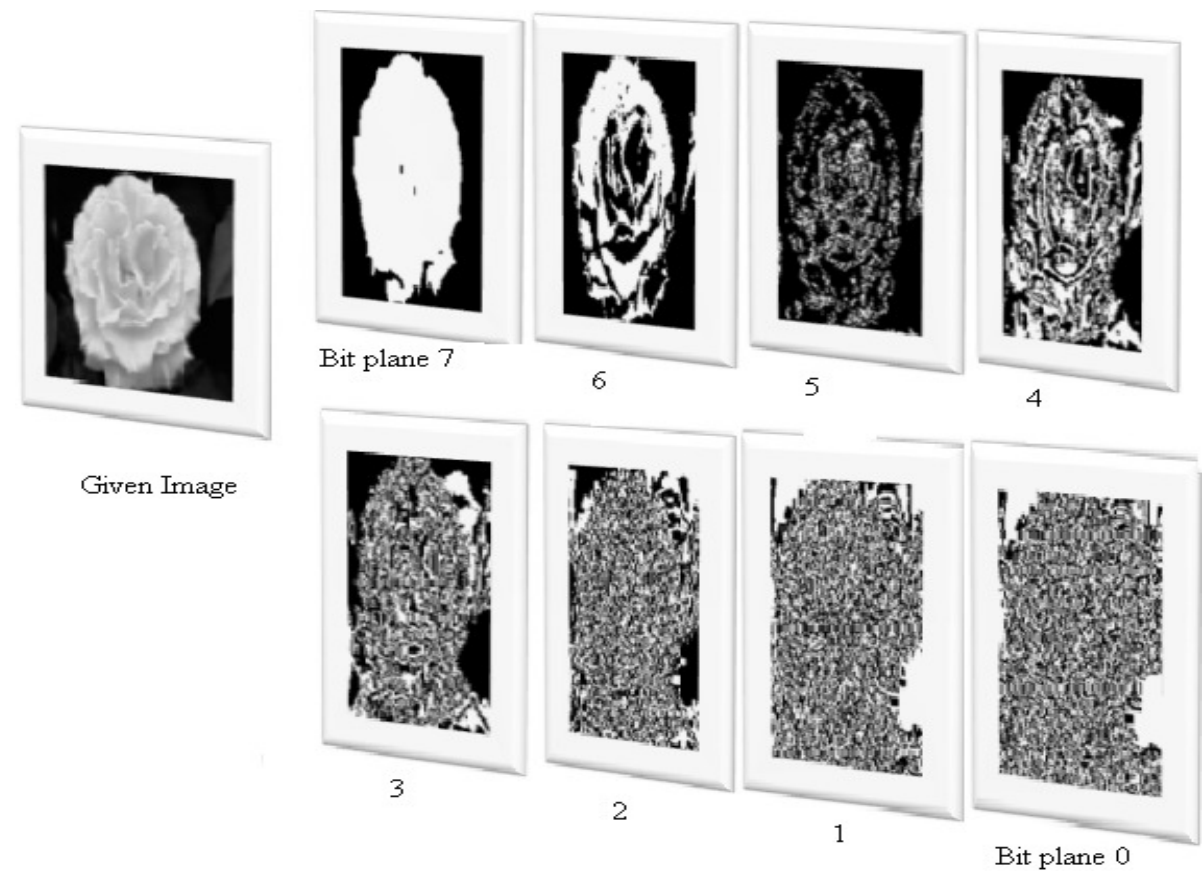

Figure 5. Gray scale image and its eight bit plane.

\section{FeATURE Vector EXTRACTION}

In this paper four different ways are used here for feature vector extraction, which is listed below,

- Feature vector calculation using single bit plane of interband average image.

- Feature vector calculation using three bit plane of color image.

- Feature vector calculation using eight bit plane of gray scale image.

- Feature vector calculation using twenty four bit plane of color image.

There for feature vector size for each method is different. It is small for the single bit plane and large for the sixteen bit planes. We are comparing the image retrieval system performance by computing the feature vector using this four methods.

\subsection{Feature Vector Extraction using Single Bit Plane of Interband Average Image}

For given color image compute the interband average image by using equation 1.Using this interband average image compute single bit plane as given in section IV (A).This bit plane is in " 1 "and " 0 "form having same size of given color image .Compute row pixel distribution \& column pixel distribution of give bit plane. The row vector is number of $1 \mathrm{~s}$ of the respective binary rows. The column vector is number of $1 \mathrm{~s}$ in respective columns. Fig. 6 is representation of sample bit plane image having 5 rows and 5 columns the row and column vector is given below. 
International Journal of Computer Science \& Information Technology (IJCSIT), Vol 3, No 3, June 2011

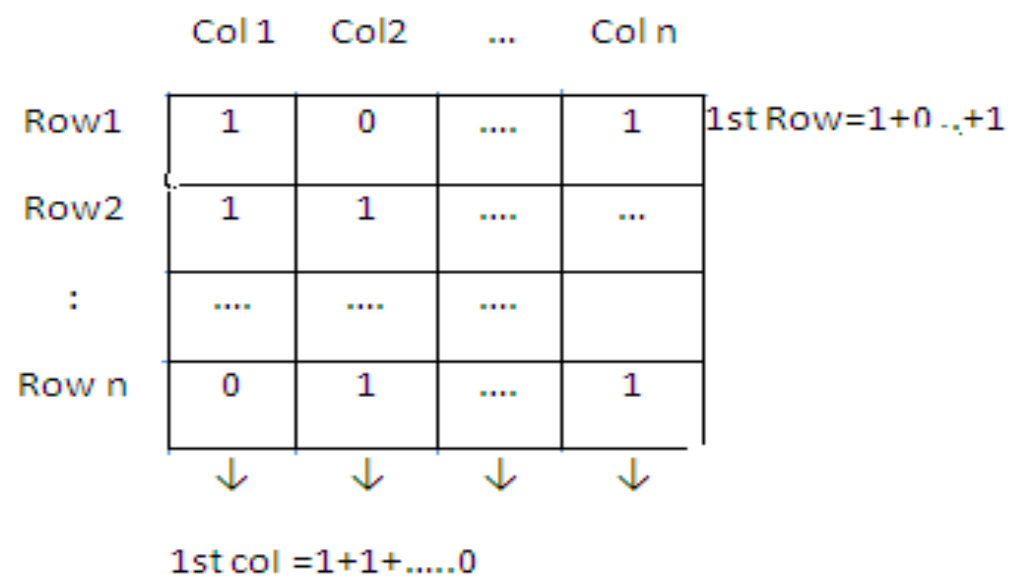

Figure 6. Calculation of row \& column pixel Distribution

After calculation of column wise and row wise pixel distribution we are getting one row and one column coefficients vector for bit pane. i.e $S B \operatorname{Pr} o w(i, 1)$ is the row wise pixel distribution and $\operatorname{SBP} \operatorname{col}(1, j)$ is the column wise pixel distribution for single bit plane. Calculate mean, Standard deviation $[1,3,12]$ and cube root of third moment of row vector and column vector using formula given in equation 11,12 and 13.Consider image size is $256 \mathrm{X} 256$.

$$
\begin{aligned}
\mu_{\text {SBProw }} & =\frac{1}{256} \sum_{i=1}^{256} \operatorname{SB} \operatorname{Prow}(i, 1) \\
\mu_{\text {SBPcol }} & =\frac{1}{256} \sum_{j=1}^{256} \operatorname{SB} \operatorname{Pcol}(1, j) \\
\sigma_{\text {SBProw }} & =\sqrt{\frac{1}{256} \sum_{i=1}^{256}\left(S B P_{\text {row }}(i, 1)-\mu_{\text {SBProw }}\right)^{2}} \\
\sigma_{\text {SBPcol }} & =\sqrt{\frac{1}{256} \sum_{j=1}^{256}\left(S B P_{\text {col }}(1, j)-\mu_{S B P \text { col }}\right)^{2}} \\
M_{3 S B P r o w} & =\sqrt[3]{\left.\frac{1}{256} \sum_{i=1}^{256} S B P_{\text {row }}(i, 1)-\mu_{\text {SBProw }}\right)^{3}} \\
M_{3 S B P c o l} & =\sqrt[3]{\frac{1}{256} \sum_{j=1}^{256}\left(S B P_{\text {col }}(1, j)-\mu_{S B P c o l}\right)^{3}}
\end{aligned}
$$


Where $\mu, \sigma$ and $M_{3}$ are the mean value, standard deviation value and third moment respectively for the row and column vector of $\operatorname{SBP}(i, j)$ image. Some times of third moment value is too large so at that time take a cube root of third moment. Now feature vector having size 6 is

$$
F V=\left[\mu_{S B \text { Prow }}, \mu_{S B P c o l}, \sigma_{S B \text { Prow }}, \sigma_{\text {SBPcol }}, M_{3 S B \text { Prow }}, M_{3 S B P c o l}\right]
$$

\subsection{Feature Vector Extraction using Three Bit Planes of Color Image}

In this case feature vector extracted from separate color component. Red, green and blue component have individual single bit plane which is given in section IV(B). Thus using this technique each color image have three bit planes. Compute row pixel distribution \& column pixel distribution of give bit planes. The row vector is number of $1 \mathrm{~s}$ of the respective binary rows. The column vector is number of $1 \mathrm{~s}$ in respective columns as shown in Fig. 6. After calculation of column wise and row wise pixel distribution for each bit plane we are getting three row and three column coefficients vector. Compute mean ,standard deviation and cube root of third moment as equations given in section V (A) for each row and column vector for each bit planes feature vector having size is 18(Six mean value, six standard deviation value and six third moment value) .

\subsection{Feature Vector Extraction using Eight Bit Planes of Gray Scale Image}

As explained in the section IV (C) for each intensity value we can compute 8-bit binary array [21][6][7]. So for gray scale image we can form 8-bit planes as shown in Fig.5.Now we have 8bit plane apply the same procedure for each bit plane which is explained in section $\mathrm{V}(\mathrm{A})$. Thus eight row vector having eight mean value, eight standard deviation value eight cube root of third moment value and eight column vector having eight mean value, eight standard deviation value ,eight cube root of third moment value. So feature vector size for using this method for eight bit plane for gray scale image is 48 .

\subsection{Feature Vector Extraction using Twenty Four Bit Planes of Color Image}

We know each intensity value have 8-bit binary array. Each image intensity have eight bit planes .So as explained in section IV(C) most of the color images are recorded in RGB color space .For each color plane have eight bit plane as shown in Fig. 7.

So for color image considering separate RGB color plane have total 24 bit planes. Now we have 24-bit planes apply the same procedure for each bit plane which is explained in section $\mathrm{V}(\mathrm{A})$.Feature vector in this case include 48 mean values of row and column vector ,48 standard deviation values of row and column vector and 48 cube root of third moment values of row and column vector for each color image. Total size of the feature vector is 144 .

\section{EXPERIMENTAL RESULTS}

\subsection{Feature Vector Matching}

When a query image is submitted by a user, we need to compute the feature vector as before and match it to the precomputed feature vector in the database. This is shown in Fig. 8. block diagram of retrieval process consist of feature extraction process, feature vector storage process and similarity measure process. The feature extraction process is based upon the following. Which the batch feature extraction and storage process as described in the following steps. 
International Journal of Computer Science \& Information Technology (IJCSIT), Vol 3, No 3, June 2011

a. Images taken one by one from the database.

b. Feature is computed using the feature extraction process.

c. Make feature vector database for given database images.

Figure 8. Feature extraction and storage process for an image collection
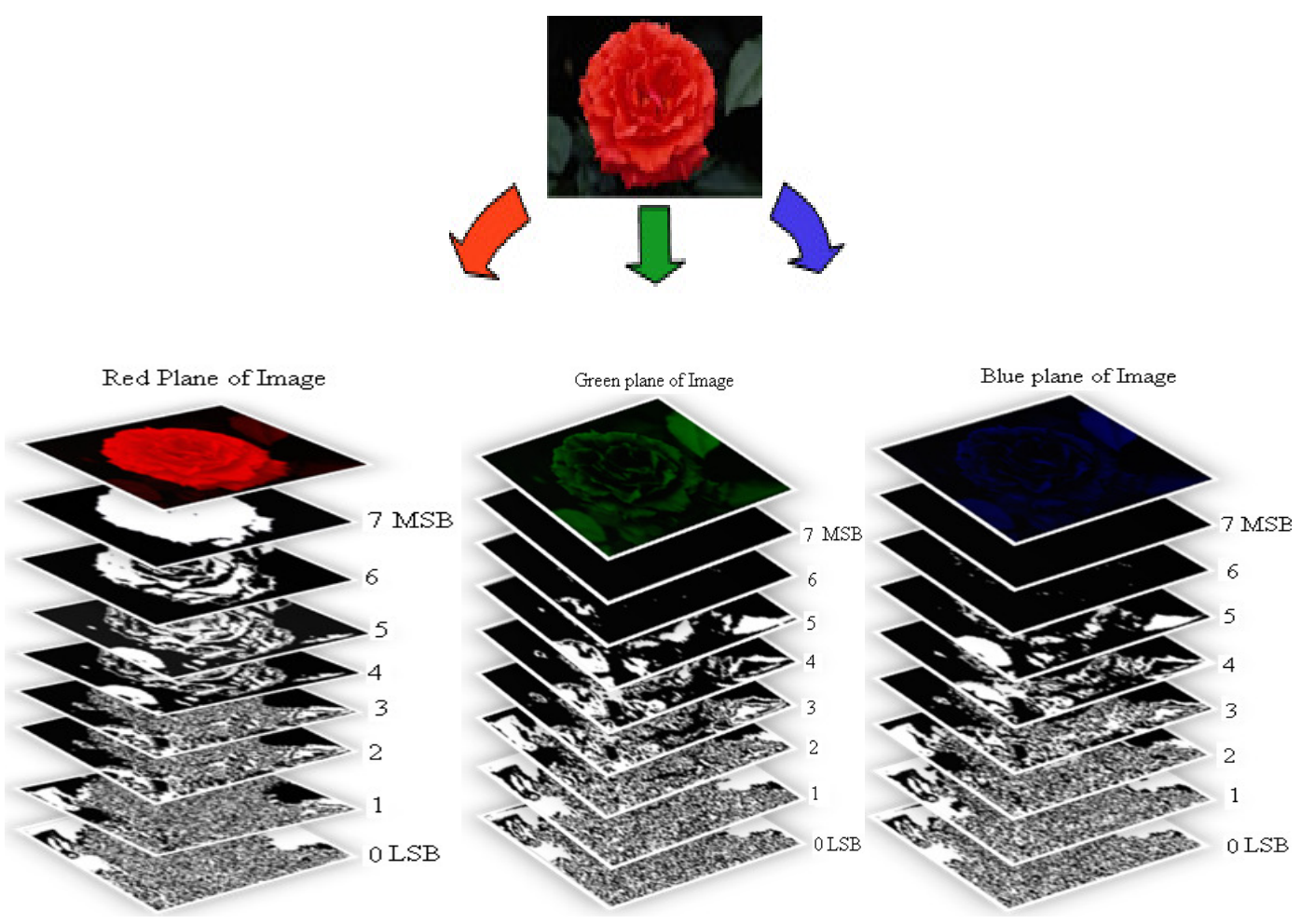

Figure 7. Color image and its red color eight bit plane, green color eight bit plane and blue color eight bit plane .

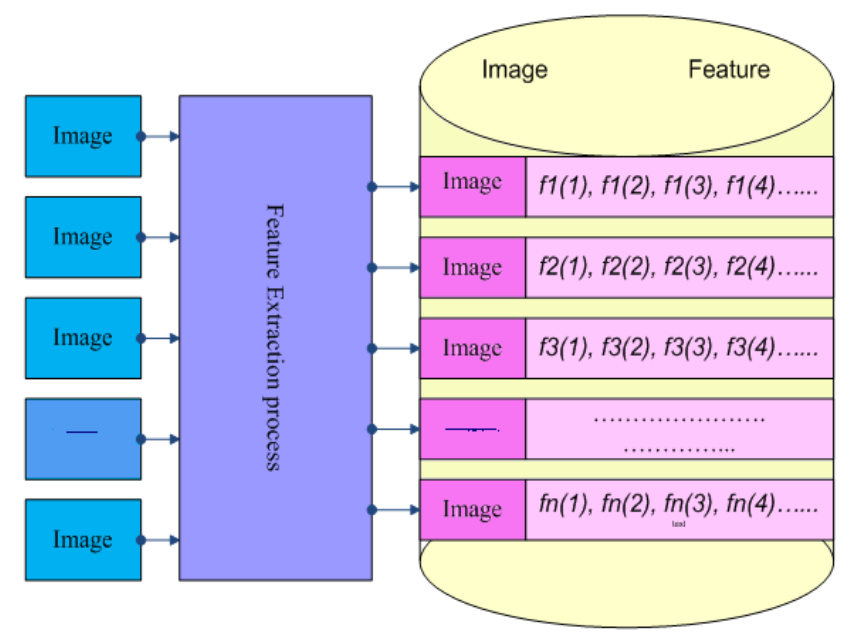

Figure 8 . Feature extraction and storage process for an image collection 
After that query image and database image matching is done using Euclidean distance. Minkowski (Euclidean distance when $\mathrm{r}=2$ ) distance is computed between each database image $\&$ query image on feature vector to find set of images falling in the class of query image.

$$
E d(Q, I)=\left(\sum_{M=0}^{M-1}\left|\mathrm{H}_{Q}-H_{I}\right|^{r}\right)^{1 / r}
$$

Where Q-Query image

I- Database image.

$\mathrm{H}_{\mathrm{Q}}$-Feature vector query image.

$\mathrm{H}_{\mathrm{I}}-$ Feature vector for database image.

M-Total no of component in feature vector.

\subsection{Performance of CBIR}

Performance of image retrieval system can be analyzed by using two parameters precision and recall. As shown in Fig.8. Testing the effectiveness of the image search engine is about testing how well can the search engine retrieve similar images to the query image and how well the system prevents the return results that are not relevant to the source at all in the user point of view.A sample query image must be selected from one of the image category in the database. When the search engine is run and the result images are returned, the user needs to count how many images are returned and how many of the returned images are similar to the query image. The first measure is called Recall. All the relevant images from the database is recall. The equation for calculating recall is given below:

$$
\text { Recall }=\frac{\text { Number_of_relevant_images_retrived(A) }}{\text { Total_number_of_relevant_images_in_database(A+D) }}
$$

The second measure is called Precision. It is accuracy of a retrieval system to present relevant as well as non relevant images from the database which is mathematically given as:

$$
\text { Precision }=\frac{\text { Number_of_relevant_images_retrived }(\mathrm{A})}{\text { Total_number_of_images_retrived }(\mathrm{A}+\mathrm{B})}
$$

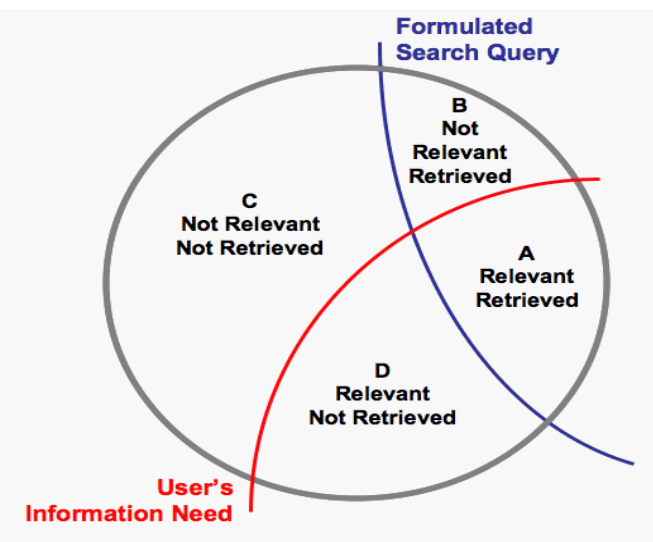

Figure 9. Evaluation of CBIR 


\subsection{Implementation and Result}

The implementation of CBIR technique is done in MATLAB 7.0 using a computer with Intel Core 2 Duo Processor T8100 (2.1GHz) and 2 GB RAM. The CBIR technique are tested on the image database of 800 variable size images includes 8 categories of animals, buses, flowers ,bikes, beaches, Historical, Mountains etc.

Sample images from each category shown in Fig.10 for 40 query images (five from each category from database) the precision and recall is calculated for proposed methods and average recall precision is plotted against category.

The average precision is calculating by using following equation 21,22 . The average precision for images belonging to the qth category (Aq) has been computed by:

$$
\bar{P}_{q}=\sum_{k \in A q} P\left(I_{K}\right) /\left|\left(A_{q}\right)\right|, q=1,2, \ldots .5
$$

Finally, the average precision is given by:

$$
\bar{P}=\sum_{q=1}^{5} \overline{P_{q}} / 5
$$

The average recall is also calculated in the same manner.

The average precision and average recall of this CBIR technique act as a important parameter to judge their performance. To determine which bit plane method is perform better in terms of precision and recall . Fig.11 show recision/recall verses image category class plots for single bit plane of interband average image .which shows overall precision and recall values for all class of the images are average. Only dinosaur mage class have recall vlue greater than $50 \%$.

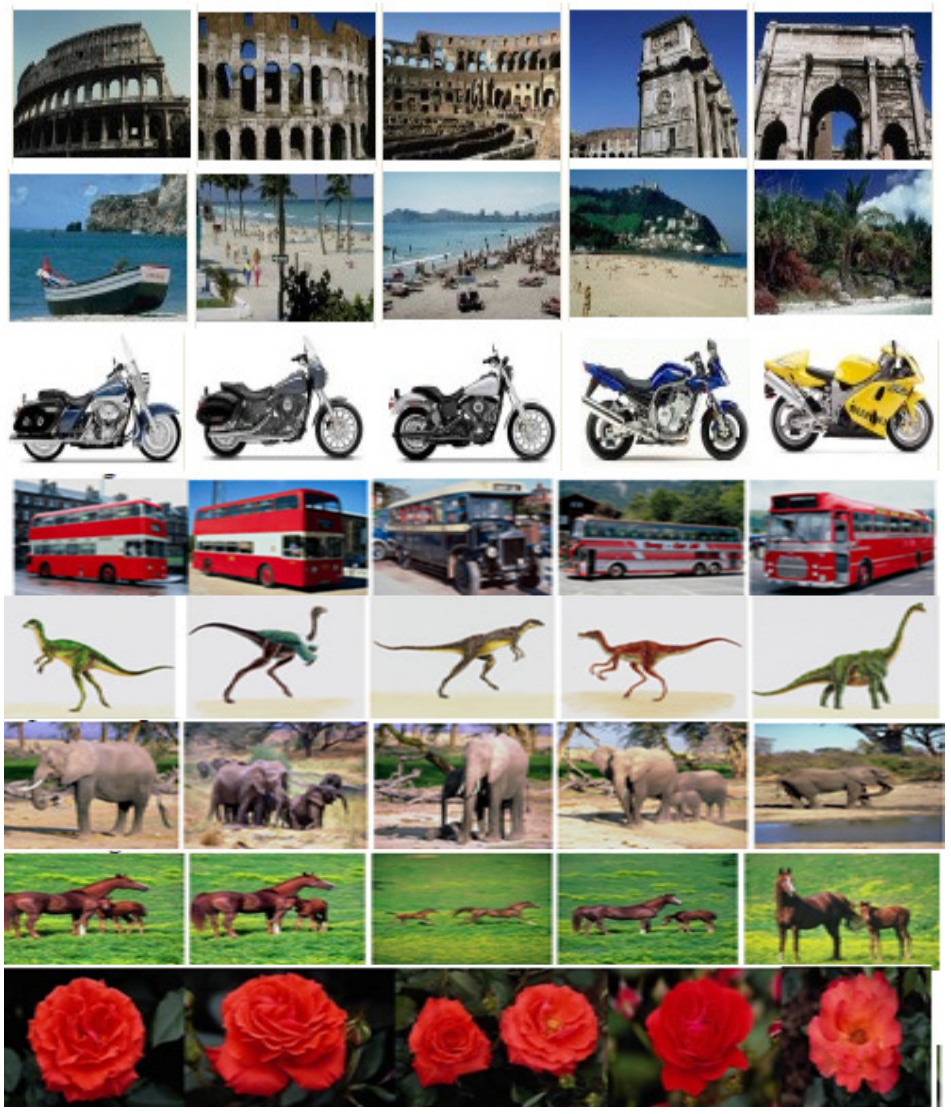

Figure 10.Sample images from database. 
International Journal of Computer Science \& Information Technology (IJCSIT), Vol 3, No 3, June 2011

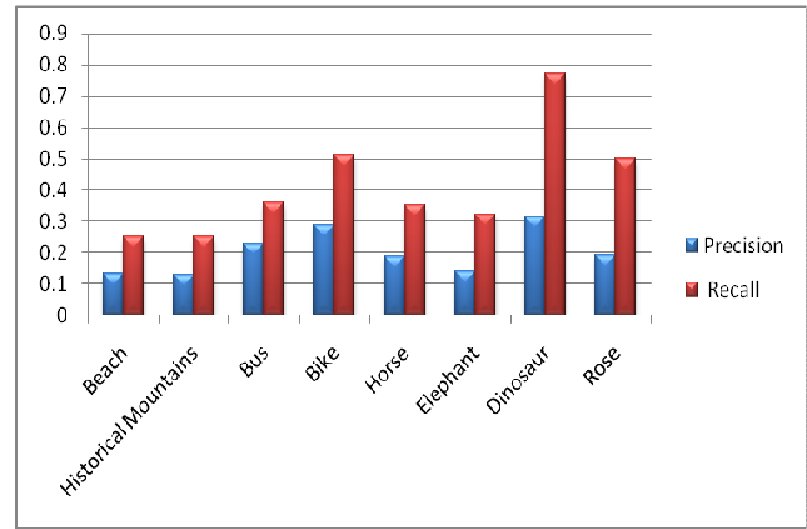

Figure 11.Average Precision and Average Recall performance for single bit plane of interband average image.

Fig.12 show precision/recall verses image category class plots for three bit plane of color image .It is observed that the recall value for some of catogaries goes on increasing

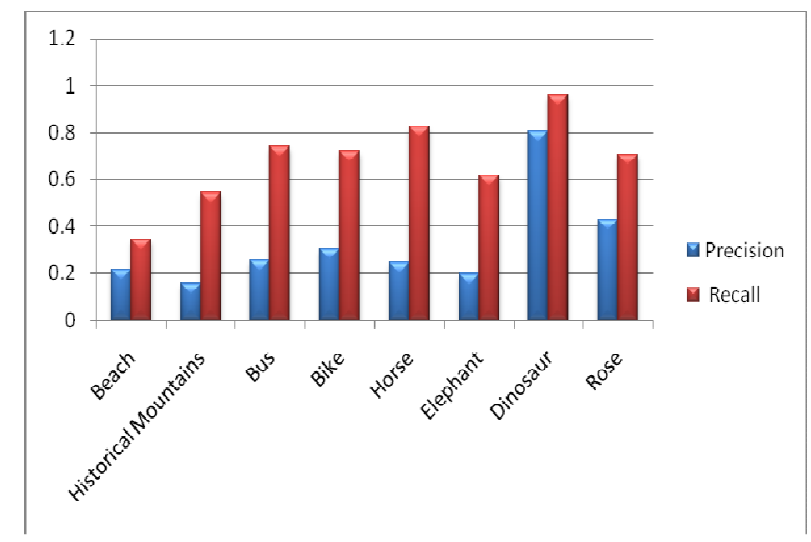

Figure 12.Average Precision and Average Recall performance for three bit planes of color image.

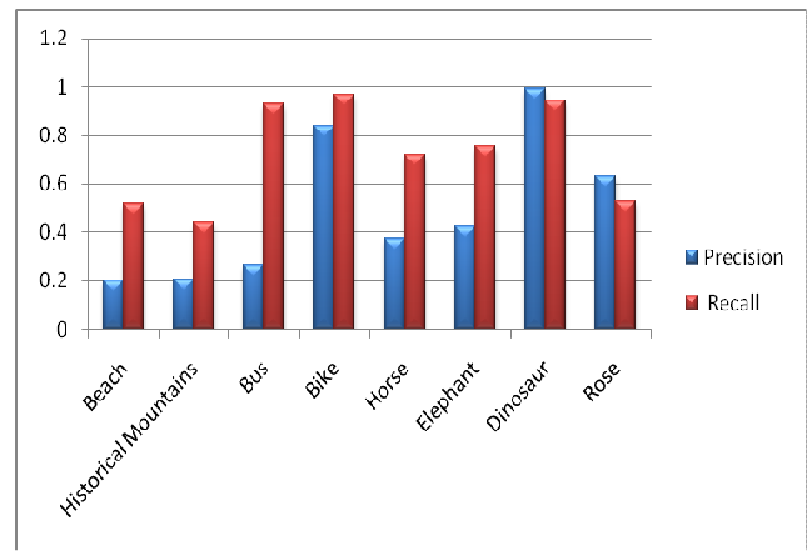

Figure 13.Average Precision and Average Recall performance for eight bit planes of gray scale image. 
but precision value is approximatly same that means there is some improvement in the recall value when we consider the separate color plane.

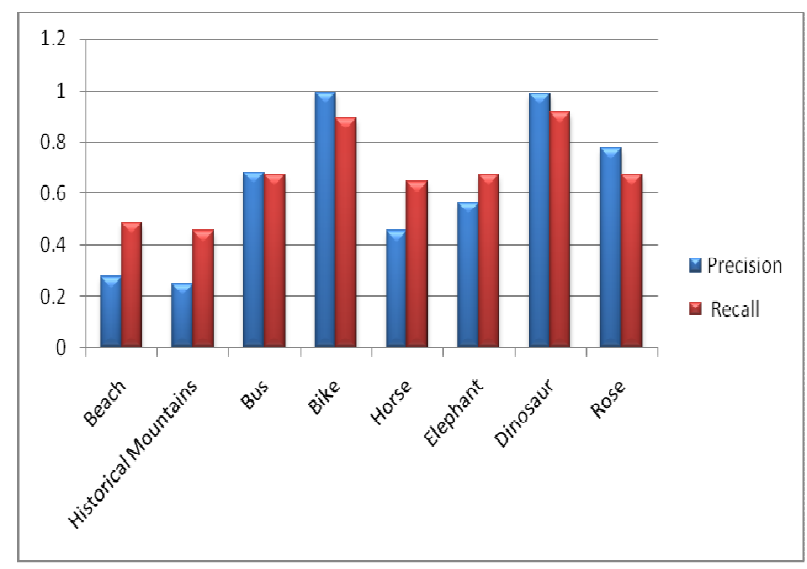

Figure 14.Average Precision and Average Recall performance for twenty four bit planes of color image.

Fig.13 show the plot for gray scale image in which the precision value also increasing with the recall in some category but $\mathrm{n}$ Fig. 14 both of the performance parameter goes on increasing. Beach and historical mountains have average precision and recall.

Table 1. Overall average precision and overall recall for four methods.

\begin{tabular}{|c|l|c|c|}
\hline Sr.No. & Feature Vector Extraction & Overall Average Precision & $\begin{array}{c}\text { Overall Average } \\
\text { Recall }\end{array}$ \\
\hline 1 & Gray scale image 1 bit plane & 0.201 & 0.413 \\
\hline 2 & Color image 3 bit plane & 0.325 & 0.681 \\
\hline 3 & Gray scale image 8 bit plane & 0.491 & 0.724 \\
\hline 4 & Color image 24 bit plane & 0.622 & 0.676 \\
\hline
\end{tabular}

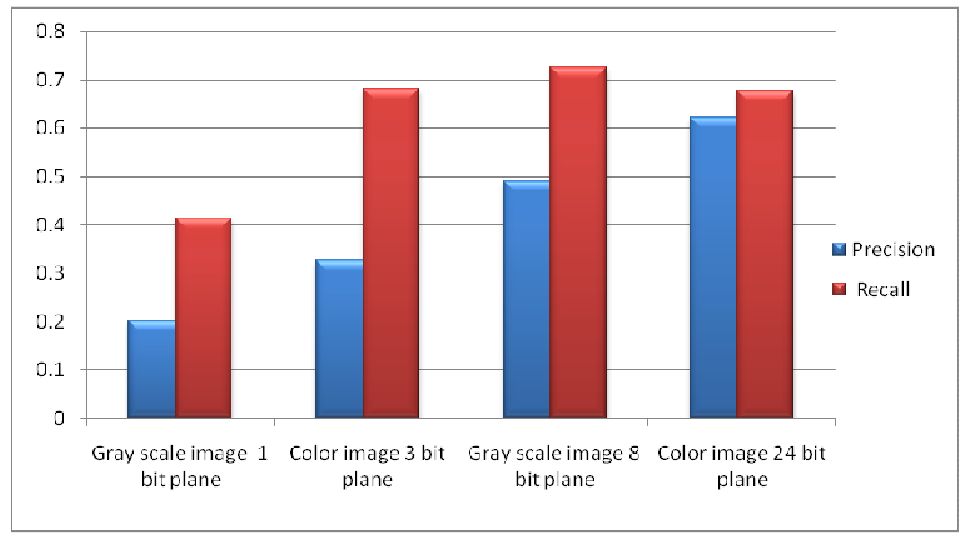

Figure 15.Overall Average Precision and overall Average Recall for proposed methods.

When we consider overall average precision and overall average recall for all methods as shown in table I and plots are shown in Fig 15.It observed that the overall average precision and overall average recall increases as the bit plane generation goes on increasing. 


\section{CONCLUSION}

We have presented a new algorithm for digital image search and retrieval in this paper. We have used mean, standard deviation and cube root of pixel distribution of image bit planes. This bit plane generations is different using mean and using binary vector for each image pixel. When we consider mean as a threshold then for interband average image only one bit plane and for color image each color plane have one bit plane. When we consider 8 bit binary vector for each pixel in the image then for gray scale image eight bit plane and for color image twenty four bit plane is to be generated. So feature vector size goes on increasing and average precision and average recall value goes on increasing. Eight bit plane and twenty four bit plane precision / recall result is good compare to the three bit plane and one bit plane.

\section{REFERENCES}

[1] NST Sai, Ravindra patil ,"Average Row and Column Vector Wavelet Transform for CBIR", Second international conference on Advance in Computer Vision and Information Technology (ACVIT2009), Aurangabad, India.

[2] NST Sai, Ravindra patil ,'New Feature Vector for Image Retrieval Walsh Coefficients”, Second international conference on Advance in Computer Vision and Information Technology (ACVIT2009), Aurangabad, India.

[3] NST Sai, Ravindra patil ,'Image Retrieval using DCT Coefficients of Pixel Distribution and Average Value of row and Column Vector "IEEE International Conference on Recent Trends in Information ,Telecommunication and Computing(ITC2009),Kochi, Kerala, India.

[4] NST Sai, Ravindra patil," Moments of Pixel Distribution of CBIR" International Conference and Workshops on Emerging Trends in Technology (ICWET2010), Mumbai, India.

[5] NST Sai, Ravindra patil ,"New Feature Vector for Image Retrieval: Sum of the Value of Histogram Bins "IEEE Conference on Advance in Computing, Control \& Telecommunication Technologies (ACT2009), Trivandrum, India.

[6] NST Sai, Ravindra patil,'Image Retrival usng Equalized Histogram Image Bins Moment” Inter national Joint Journal Conference in Engneering ,IJJCE,2010,Trivandrum,India.

[7] R.C. Gonzalez, and R.E. Woods, Digital Image Processing 2nd ed.,Prentice Hall, Inc., New Jersey, 2002.

[8] K.C. Ting,D.B.L.Bong,Y.C.Wang,"Performance Analysis of Single and Combined Bit-Planes Feature Extraction for Recognition in Face Expression Database ", Proceedings of the International Conference on Computer and Communication Engineering 2008,May 13-15, 2008 Kuala Lumpur, Malaysia .

[9] Guoping Qiu, “Colour Image Indexing Using BTC”, IEEE Transition on Image Processing, vol. No 12,.Janauary 2003.

[10] Pdamshree Suresh,RMD Sundaram,Aravindhan Arumugam,” Feature Extraction in Compressed Domain for Content Based Image Retrieval ",International Conference on Advanced Computer Theory and Engineering.2008.

[11] M. Flickner, H. Sawhney, W. Niblack, J. Ashley, Q. Huang, B. Dom, M. Gorkani, J. Hafner, D. Lee, D. Petkovic, D. Steele, and P. Yanker, "Query by Image and Video Content: The QBIC System", IEEE Computer, 28(9):23-32, Sept. 1995.

[12] A. W. M. Smeulders, M. Worring, S. Santini, A. Gupta, and R. Jain, "Content-based image retrieval at the end of the early years," IEEE Transaction on Pattern Analysis and Machine Intelligence (PAMI), 22(12):1349-1380, Dec. 2000.

[13] K. Hirata and T. Kato, a Query by Visual Example," Advances in Database Technology EDBT '92, Third Int'l Conf. Extending Database Technology, 1992.

[14] W.Y. Ma and B.S. Manjunath, "Pictorial Queries: Combining Feature Extraction with Database Search," Technical Report 18, Dept. of Electrical Eng., Univ. of California at Santa Barbara, 1994.

[15] W.Y. Ma and B.S. Manjunath, "Pictorial Queries: Combining Feature Extraction with Database Search," Technical Report 18, Dept. of Electrical Eng., Univ. of California at Santa Barbara, 1994.

[16] A. Gupta and R. Jain, ${ }^{a}$ Visual Information Retrieval," Comm. ACM, vol. 40, no. 5, 1997.

[17] C.E. Jacobs, A. Finkelstein, and D.H. Salesin, "Fast Multiresolution Image Querying," Proc. SIGGRAPH 95, 1995.

[18] W.J.Z. Wang, G. Wiederhold, O. Firschein, and S.X. Wei, "Wavelet Based Image Indexing Techniques with Partial Sketch Retrieval Capability,” J. Digital Libraries, 1997.

[19] Seung Jun-Lee, Yong-Hwan Lee, Hyochang Ahn, Sang Burm Rhee, "Color image descriptor using wavelet correlogram," The 23rd international conference on Circuits/systems, computers and communication, 2008 . 
[20] M.Mohammed Sathik,"Feature Extracton on ColorED x-Ray Images by Bit-plane Slicing Technique",International Journal of Engineering Science and Technology Vol. 2(7), 2010, 28202824.

[21] Govind Haldankar, Atul Tikare and Jayprabha Patil, "Converting Gray Scale Image to Color Image" in Proceedings of SPIT-IEEE Colloquium and International Conference, Mumbai, India, Vol. 1, 189.

[22] Pratt W.K., Digital image processing, A Wiley Interscience Publication, 1991.

[23] N.Ravia Shabnam Parveen, Dr. M.Mohamed Sathik, "Feature Extraction by Bit Plane Slicing Technique", in International Journal of Computing, Communication and Information System, Volume 1.

[24] M. K. Mandal, T. Aboulnasr, and S. Panchanathan,, "Image Indexing Using Moments and Wavelets", IEEE Transactions on Consumer Electronics, Vol. 42, No. 3, August 1996.

[25] A Gupata and R. Jain, "Visual Information Retrieval," Comman. ACM, vol.40, no.5, 70- 79, 1997.

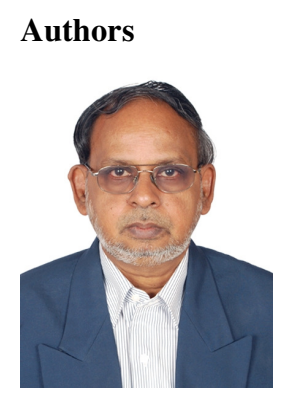

NST Sai obtained PhD in 1985 from IIT Delhi. He served as faculty at BITS, Pilani during 1985-89 and RAIT, a Mumbai University Affiliate 1990 - 2001 before joining Tech Mahindra Limited in 2001. He has been a mentor and supervisor for several students of BE/MS/MBA programs pursuing from BITS, Pilani, University of Mumbai, University of Pune and NMIMS university over the past several years. He has a total experience of over 25 years. He has several pioneering initiatives to his credit both in technical and behavioral domains. His areas of technical interest include Multimedia Computing, Robotics and Signal processing. His areas of interest in behavioral domain include human resource management, training, skill development and work integrated learning. Currently he is facilitating the pursuit of higher education programs of employees and program managing the delivery of the same in collaboration with various universities.

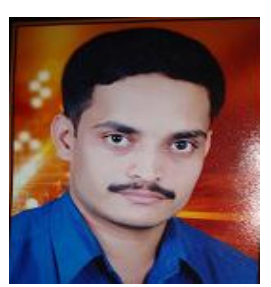

Ravindra C. Patil has received M.E (degree in Electronics Engineering from University of Mumbai. He is currently working as a faculty member at Department of Computer Engineering at TCET affiliated to University of Mumbai and pursuing the doctoral research at MPSTME affiliated to NMIMS Deemed University, Mumbai. His area of interest is CBIR techniques in Image Processing. 